\title{
Asynchronous Multi-Objective Optimisation in Unreliable Distributed Environments
}

\author{
Andrew Lewis ${ }^{1}$ and Sanaz Mostaghim ${ }^{2}$ and Ian Scriven ${ }^{3}$
}

\begin{abstract}
This chapter examines the performance characteristics of both asynchronous and synchronous parallel particle swarm optimisation algorithms in heterogeneous, fault-prone environments. The chapter starts with a simple parallelisation paradigm, the Master-Slave model using Multi-Objective Particle Swarm Optimisation (MOPSO) in a heterogeneous environment. Extending the investigation to general, distributed environments, algorithm convergence is measured as a function of both iterations completed and time elapsed. Asynchronous particle updates are shown to perform comparably to synchronous updates in fault-free environments. When faults are introduced, the synchronous update method is shown to suffer significant performance drops, suggesting that at least partly asynchronous algorithms should be used in real-world environments. Finally, the issue of how to utilise newly available nodes, as well as the loss of existing nodes, is considered and two methods of generating new particles during algorithm execution are investigated.
\end{abstract}

Institute for Integrated and Intelligent Systems

Griffith University

Brisbane

Queensland

Australia

A. Lewisegriffith.edu.au

Institute AIFB

University of Karlsruhe

Germany

sanaz.mostaghimekit.edu

School of Engineering

Griffith University

Brisbane

Queensland

Australia

Ian.Scrivenestudent.griffith.edu.au 


\section{Introduction}

Optimisation of solutions using computational models is becoming an increasingly common practice in engineering design and scientific investigations. It has been applied to a wide variety of problems, from biomechanics [26] to avionics [44] and the demand is increasingly for the simultaneous optimisation of several, possibly competing objectives. Such real-world problems usually require complex, timeconsuming computer simulations to solve potential solutions and so parallelisation of optimisation algorithms is a practical necessity. Originally confined to dedicated, parallel computing clusters or special-purpose parallel computers, the emergence of grid computing as a common approach to the provision of computational capacity [1] has dictated the development of optimisation algorithms capable of efficient and effective performance in potentially unreliable distributed environments. Such algorithms can also be applied effectively to utilise small-scale, ad hoc grids of networked computers, bringing the benefits of computational optimisation within the reach of small to medium enterprises.

Several Parallel Evolutionary Algorithms have been studied in the literature (e.g., $[3,6,43,16,11,10]$ ). From these studies three main paradigms emerge: the Island model, Master-Slave model and Diffusion model. The focus of this chapter is on the Master-Slave model, a straight-forward method and perhaps the simplest, where a single processor maintains the optimisation task and uses the other processors for objective function evaluations.

We start by studying parallelisation of multi-objective optimisation (MO) algorithms using the Master-Slave model. The Master-Slave model is the simplest parallelisation paradigm when working in a homogeneous environment, and has been widely implemented in such. However, to our knowledge little has been done on the combination of multi-objective optimisation, heterogeneous resources and the Master-Slave model. Comparing multi-objective algorithm with their single objective counterparts there is a major difference. A multi-objective EA (MOEA) must evaluate not just enough trial solutions for effective search of the parameter space, but sufficient to allow determination of the relative fitness against the multiple objectives. In a Master-Slave model, the master processor has to wait for all of the evaluations from the other processors. Then it can apply a ranking method to the solutions to find the non-dominated front and continue the optimisation. In a heterogeneous environment, the waiting time might be very long. In some cases, the fast processors can deliver twice the evaluations of a slow one, in a given period of time. In this work, we study a new algorithm which utilises all of the computing resources, from the slow to the very fast (as in a normal grid).

Solving multi-objective problems on heterogeneous systems has a number of challenges:

- Dealing with heterogeneous resources, the aim is to use all of the computing resources but at the same time to be efficient in time, i.e., the master node must make use of all of the available function evaluations in every time step. Here, the 
master node encounters a trade-off between waiting for the slowest processor or continuing the optimisation based on the available solutions.

- The output of multi-objective optimisation problems is usually a set of socalled Pareto-optimal solutions. Most multi-objective evolutionary algorithms (MOEAs) approximate these solutions by finding a set of non-dominated solutions. In every generation, a typical MOEA method applies a ranking-based method to the entire population to evaluate the solutions.

- Efficient and effective exploration of the search space is another important issue, in common with most practical applications of optimisation. The Master-Slave model is typically used to solve computationally expensive problems. The main task would be to employ a reasonable exploration technique while minimising the computational cost.

One drawback of the Master-Slave model is the communication overhead between processors. Since the simulation models considered in real-world engineering design problems are typically complex and computationally expensive, the computation time will usually outweigh communication time, so this factor can safely be ignored.

Particle Swarm Optimisation (PSO) is fast being established as an efficient, parallel optimisation technique for both single and multiple objective design problems. In Multi-Objective Particle Swarm Optimisation (MOPSO), each particle (individual) follows its own best position and the position of a global guide. Hence, in the parallelisation scheme, it is enough to find a guide for a particular particle to improve that particle's position. This is an advantage compared to other MOEA techniques such as SPEA2 [48] or NSGAII [17]. In SPEA2, all of the individuals are compared to each other and are given a strength and in NSGAII a ranking method finds different non-dominated fronts. In these methods, the best evaluation is based on the entire population.

In distributed systems, different kinds of failures can happen. Some typical failures among the others are: nodes may get overloaded, fail or loose their connection to the other nodes. In this chapter, we also address optimisation using such unreliable environments. We name the new approach Parallel Asynchronous Particle Swarm Optimisation (PAPSO) and we examine the effects of asynchronous updates in PAPSO algorithms in fault-prone environments, and compare the convergence characteristics of Parallel Synchronous Particle Swarm Optimisation (PSPSO) [37] and PAPSO algorithms against both iteration count and wall-clock execution time.

Apart from investigating the failures in the system, we address the aspect of having new nodes in the available system, known as churn. We use the new nodes for improving the quality of the approximated front obtained by MOPSO.

This chapter is organised as follows. In the following we introduce the test functions used throughout the chapter. In the next sections (2 and 3), we study synchronous and asynchronous parallel multi-objective particle swarm optimisation. Sections 4 and 5 are about unreliable environments and parallel MOPSO methods working on such environments. In Section 6, we address the churn issue in our approach and propose two new methodologies for covering and extending the edges of the obtained approximated front. Section 7 concludes the chapter. 
Table 1 Test functions

\begin{tabular}{lll}
\hline Test & Function & Constraints \\
\hline ZDT1 & $g\left(x_{2}, \cdots, x_{n}\right)=1+9\left(\sum_{i=2}^{n} x_{i}\right) /(n-1)$ & $x_{i} \in[0,1]$ \\
& $h\left(f_{1}, g\right)=1-\sqrt{f_{1} / g}$ & $n=30$ \\
& $f_{1}\left(x_{1}\right)=x_{1}$ & $i=1,2, \ldots, n$ \\
& $f_{2}(\mathbf{x})=g\left(x_{2}, \cdots, x_{n}\right) \cdot h\left(f_{1}, g\right)$ & \\
ZDT2 & $g\left(x_{2}, \cdots, x_{n}\right)=1+9\left(\sum_{i=2}^{n} x_{i}\right) /(n-1)$ & $x_{i} \in[0,1]$ \\
& $h\left(f_{1}, g\right)=1-\left(f_{1} / g\right)^{2}$ & $n=30$ \\
& $f_{1}\left(x_{1}\right)=x_{1}$ & $i=1,2, \ldots, n$ \\
& $f_{2}(\mathbf{x})=g\left(x_{2}, \cdots, x_{n}\right) \cdot h\left(f_{1}, g\right)$ & \\
ZDT3 & $g\left(x_{2}, \cdots, x_{n}\right)=1+9\left(\sum_{i=2}^{n} x_{i}\right) /(n-1)$ & $x_{i} \in[0,1]$ \\
& $h\left(f_{1}, g\right)=1-\sqrt{f_{1} / g}-\left(f_{1} / g\right) \sin \left(10 \pi f_{1}\right)$ & $n=30$ \\
& $f_{1}\left(x_{1}\right)=x_{1}$ & $i=1,2, \ldots, n$ \\
& $f_{2}(\mathbf{x})=g\left(x_{2}, \cdots, x_{n}\right) \cdot h\left(f_{1}, g\right)+1$ & $n=10$ \\
& $f_{1}(\mathbf{x})=1-\exp \left(-\sum_{i}\left(x_{i}-\frac{1}{\sqrt{n}}\right)^{2}\right)$ & $x_{i} \in[-4,4]$ \\
FF & $f_{2}(\mathbf{x})=1-\exp \left(-\sum_{i}\left(x_{i}+\frac{1}{\sqrt{n}}\right)^{2}\right)$ & \\
DTLZ & $f_{1}(\mathbf{x})=\left(1+g\left(x_{M}\right)\right) \cos \left(x_{1} \pi / 2\right) \cos \left(x_{2} \pi / 2\right)$ & $x_{i} \in[0,1]$ \\
& $f_{2}(\mathbf{x})=\left(1+g\left(x_{M}\right)\right) \cos \left(x_{1} \pi / 2\right) \sin \left(x_{2} \pi / 2\right)$ & $n=8$ \\
& $f_{3}(\mathbf{x})=\left(1+g\left(x_{M}\right)\right) \sin \left(x_{1} \pi / 2\right)$ & \\
& $g\left(x_{M}\right)=\sum_{i=3}^{8}\left(x_{i}-0.5\right)^{2}$ & \\
\hline
\end{tabular}

\subsection{Test Functions}

For the experiments in this chapter, some standard test functions from the multiobjective optimisation literature are used. These test functions are two- and threeobjective optimisation problems selected from $[48,18]$, as shown in Table 1.

\section{Master-Slave Model of Parallelisation}

The simplest parallelisation paradigm for optimisation is the Master-Slave model. This model is aimed at distributing the (objective function) evaluation of the individuals on several slave computing resources while a master resource executes the optimisation procedure. The master node can also be used for evaluations.

This model is of great benefit when the function evaluations are very expensive. It is very natural to use parallel computers to solve expensive functions but care must be taken that the algorithm searches efficiently. In addition, a key issue is that in many situations the computing resources are heterogeneous. In the Master-Slave model, the central computing resource (master node) has to gather information from all of the resources and perform the optimisation steps (such as ranking etc.) based on the solutions obtained. However, waiting for the slowest processor may take a long time.

The main questions when solving problems on heterogeneous computing resources therefore are: 


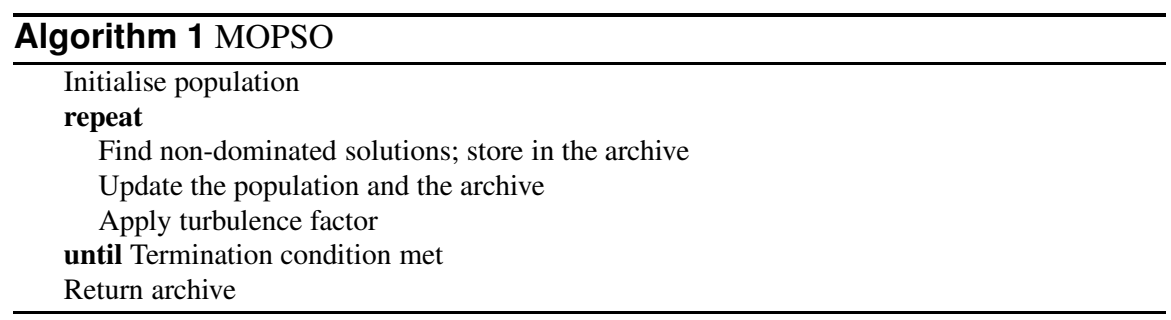

1. how to efficiently search the space and

2. how to use all of the resources so that none of them remains idle.

\section{Parallel Multi-Objective Particle Swarm}

A typical MOPSO method is shown in Algorithm 1. It starts with a random population of solutions (also called particles). Every particle $i$ has a position in the search space and a velocity, denoted by the vectors $\mathbf{x}_{i}$ and $\mathbf{v}_{i}$, respectively. The nondominated particles are stored in an archive and the population is updated based on the positions of the global best particles $P_{\text {global }}$ and their own personal memories $P_{\text {Best }}$ :

$$
\begin{gathered}
\mathbf{x}_{i}(t+1)=\mathbf{x}_{i}(t)+\mathbf{v}_{i}(t) \\
\mathbf{v}_{i}(t)=w \mathbf{v}_{i}(t-1)+R_{1}\left(P_{\text {Best }}-\mathbf{x}_{i}(t-1)\right)+R_{2}\left(P_{\text {global }}-\mathbf{x}_{i}(t-1)\right)
\end{gathered}
$$

where $w, R_{1}$ and $R_{2}$ denote the inertia weight and control parameters. In MOPSO, selecting the best personal position and the global positions has a great impact on the quality of the solutions [5, 29]. The global best is usually selected from the archive. To avoid local optima in MOPSO, a percentage of the particles are randomly selected and moved in order to explore the search space. This is implemented using a turbulence factor. MOPSO is iteratively continued until a stopping criterion, such as a certain number of iterations or evaluations, is met. A very good survey about MOPSO can be found in [35].

Parallelising MOPSO based on the Master-Slave model for a set of homogeneous resources can be easily achieved. Algorithm 2 shows a (synchronous) Parallel MOPSO on an homogeneous set of resources. Since the computing resources are homogeneous it is safe to assume the results from slave nodes will be returned to the master node in a timely and synchronised manner, provided the computing resources and interconnection network are dedicated to the optimisation task and free of failures. In every iteration, the master node distributes the evaluation tasks and waits for all of the resources to finish.

However, the main issue in dealing with heterogeneous (asynchronous) resources is that in the worst case of having only one slow computing resource, almost all of the computing resources remain idle while the master node waits for the slowest one. Here, we want to make use of all of the computing resources in a way that, as soon as 


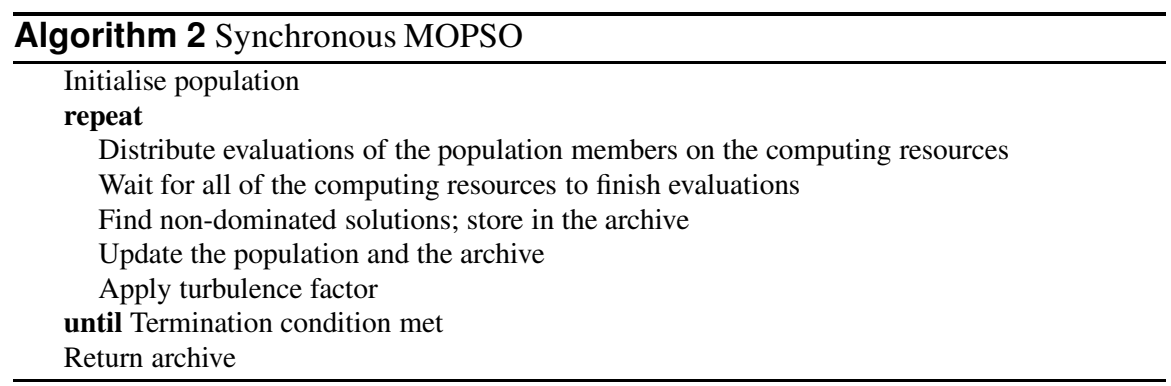

a reasonable amount of resources have completed evaluations, the master resource continues optimising without further waiting. This is not to imply the results of the slow computers are not also of interest as they also contain information about the search space. The information from them will be incorporated when it arrives at a later stage.

The first step in parallelisation on a heterogeneous set of resources is to define a good set of initial particles and send them to the slave nodes for evaluation. Usually in MOPSO a random set is used; here we propose to use a set of random particles defined by a recursive Gap Search (GS) Method as explained below. While use of Gap Search is peripheral to the investigation of asynchronous update in the MOPSO algorithm, it is a novel approach to satisfying the goal of efficient search of the parameter space.

\subsection{Exploration Using Recursive Gap Search Method}

For searching the least explored regions of a search space, Gap Search (GS) algorithm has been proposed which is very similar to the Binary Search [23]. GS is a recursive search algorithm which finds the large gaps in the search space and samples solutions in those areas where the binary search employs a successive search in most promising areas in the search space. In GS, the search is started by selecting a random point in the search space. Then the largest empty region is found in the space by computing the distances between the first point and the boundaries of the search space. Figure 1 (a) shows an example for a two dimensional space. The next point is randomly selected in that empty region (Figure 1 (b)). This is an iterative method and can be performed for any desired number of solutions. These solutions are usually stored in a list.

One disadvantage of GS is that selecting a point from a large list of solutions requires a relatively high computation time, particularly for spaces of high $(>20)$ dimensionality. In the cases at which this study is aimed, it is assumed the time taken for objective function evaluations will dominate. 
(a)

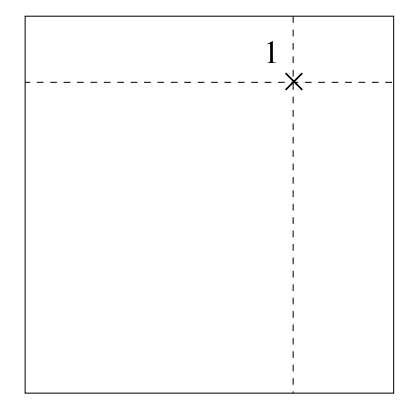

(b)

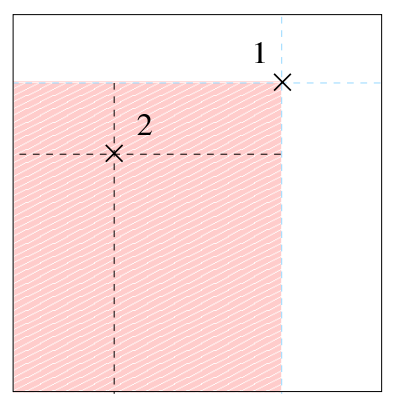

Fig. 1 An example of the Gap Search Method in a two dimensional space. In (a) one point is randomly selected in the search space. The next point is selected in the most empty region defined in (b). (c) 2008 IEEE

\subsection{Parallel MOPSO on Heterogeneous Resources}

We propose a MOPSO which starts with an initial set of solutions based on the recursive Gap Search method. These selected solutions are stored in a list and are sent to the slave processors for evaluation. The master node waits for $N_{s}\left(1 \leq N_{s} \leq N\right)$ processors where $N$ is the total number of available processors. After receiving those evaluations, the non-dominated solutions are stored in the archive and the master node sends new evaluation jobs to the $N_{s}$ processors. Note that this differs from a synchronous algorithm in that the master node is determining Pareto-dominance of solutions based on incomplete information. A synchronous MOPSO waits for all solution evaluations to be returned before processing them.

Before sending the parameter sets, the master node qualifies the evaluation of the processors. If the solution found by a processor is:

- dominated by one of the archive members: the master processor selects a proper global guide from the archive and updates the position of the corresponding particle based on MOPSO. Then the master processor sends the new position to the slave processor for evaluation.

- not dominated by any other archive member: the master processor gives the task of exploration to the corresponding processor. Based on the Gap Search Method, a proper random position in an unexplored region in the space is selected and is sent to the slave processor for evaluation.

The exploration task done by GS replaces the turbulence factor of the original MOPSO. The population members are stored in the Gap List over the generations. Algorithm 3 shows the parallel MOPSO on heterogeneous resources. 


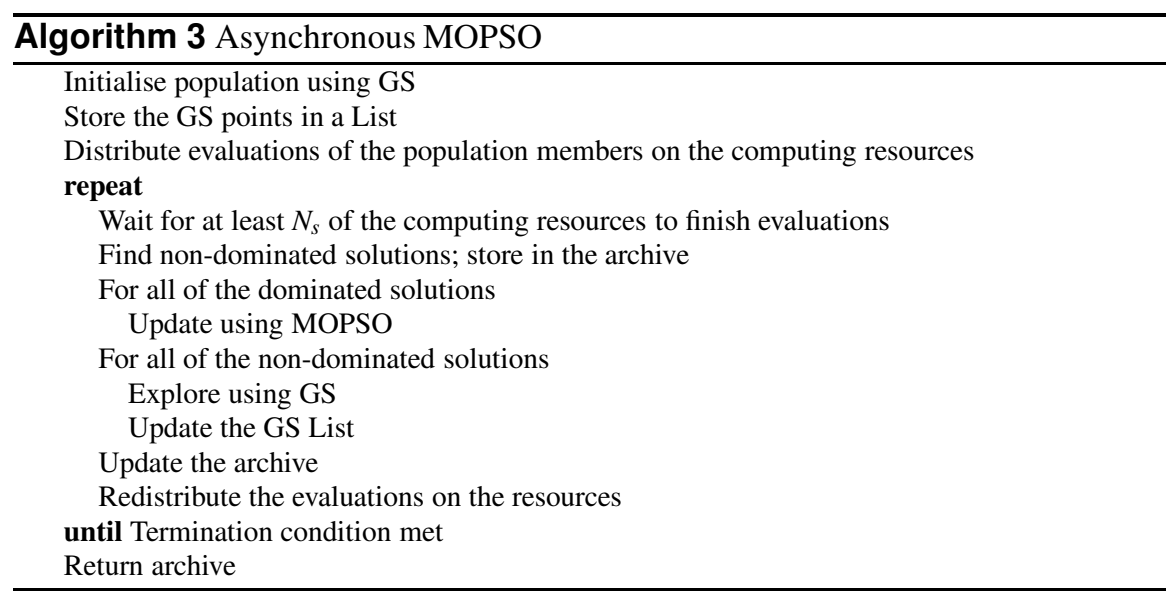

\subsection{Discussion}

In this algorithm, the parameter $N_{s}$, the least number of processors to wait for, must be known in advance. In fact, this parameter depends on the heterogeneous resources and the estimated load on them. It can also change during the iterations.

In the proposed Parallel MOPSO, we do not use a turbulence factor. Instead, we apply an intentional exploration to the non-dominated solutions using the Gap Search method. The reason is that the non-dominated solutions cannot be significantly improved by the conventional MOPSO as they constitute the global best particles from which guides are drawn. Therefore, their positions are stored in the archive and we assign them a new position based on the Gap Search. On the other hand, the position of the dominated particles can be improved by the guidance of the non-dominated ones and therefore the MOPSO method can be used effectively.

Also, in this algorithm we not only store the non-dominated solutions in the archive but we also store all the population members (explored by GS) in a list. In fact, the list is very useful to obtain knowledge about the unexplored regions, in contrast to the idea of selecting any random position in parameter space using the turbulence factor. It must be mentioned that the size of the list has a great impact on the computation time. Here, we assume that the function evaluations require a relatively high computation time and that there is a limited number of solutions in the lists so that the computation time of finding the unexplored regions in the list can be neglected.

\subsection{Experiments}

The major goal of the experiments was to evaluate the quality of the solutions when running the system for a certain time and adding waiting time (execution hetero- 


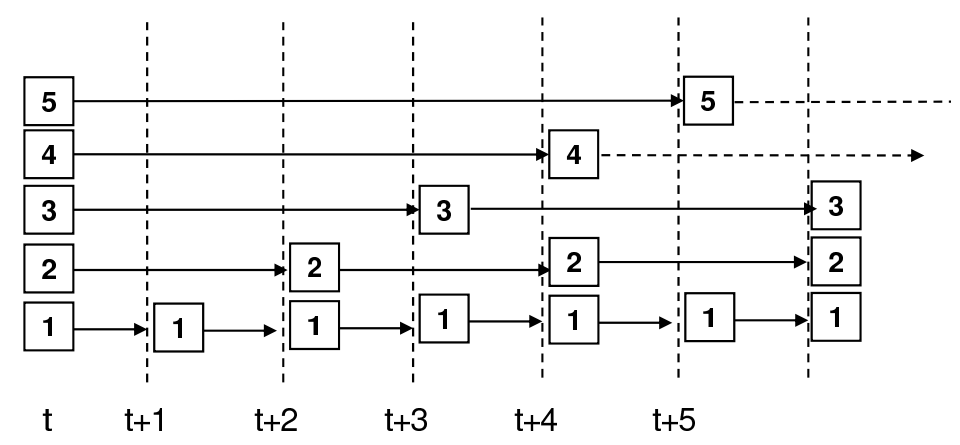

Fig. 2 Five different resources are illustrated in terms of speed. The resource type 1 is five times faster than the resource type 5. Resource types are shown in the Boxes.

geneity) to the resources. The system was analysed by adding waiting time to the fast resources to wait for a certain percentage of the slow ones. The experiments were performed on a simulation environment containing 100 resources with 5 different computation speeds. The simulation was based on a real scenario in a typical grid. Figure 2 illustrates 5 different kinds of available resources. There are 3, 3, 20, 43, and 31 number of type 1 (very fast), type 2, type 3, type 4, and type 5 (very slow) resources, respectively.

The test function FF was selected from Table 1 for these experiments. The quality of the solutions was computed by the hypervolume metric [48] averaged over 20 runs.

The asynchronous MOPSO described, an extension of the algorithm from Mostaghim and Teich [29], was executed on the master node using 100 particles. The total time $T_{\text {total }}$ was set to be 120 , meaning the fast resources were able to evaluate 120 particles.

The main archive stored in the master node was set to be empty initially (for the first run). Parameter settings were selected so that the first runs of the 100 resources included only a random sampling of the search space until the fast processors finished their evaluations and updated the archive.

Figure 3 shows the quality of the archive members over time. Different plots illustrate the quality if the resources that complete wait for $j$ other resources to finish and update the archive $(j=0,10,20,30,40,50)$.

We observe that, if the resources do not wait, the results are worse than if they wait for at least 10 to 20 percent of the resources. This result is to be expected as, in multi-objective optimisation, the results depend on each other through the dominance relation. If the resources wait (in this case) for up to 20 percent, they achieve a better quality than if they do not wait. In fact, in every step of optimisation it is better to have enough evaluations to find more dominated and non-dominated solutions. Hence waiting for a time has the advantage of receiving more solutions. This leads to a better direction in the optimisation than not waiting. However, waiting for 


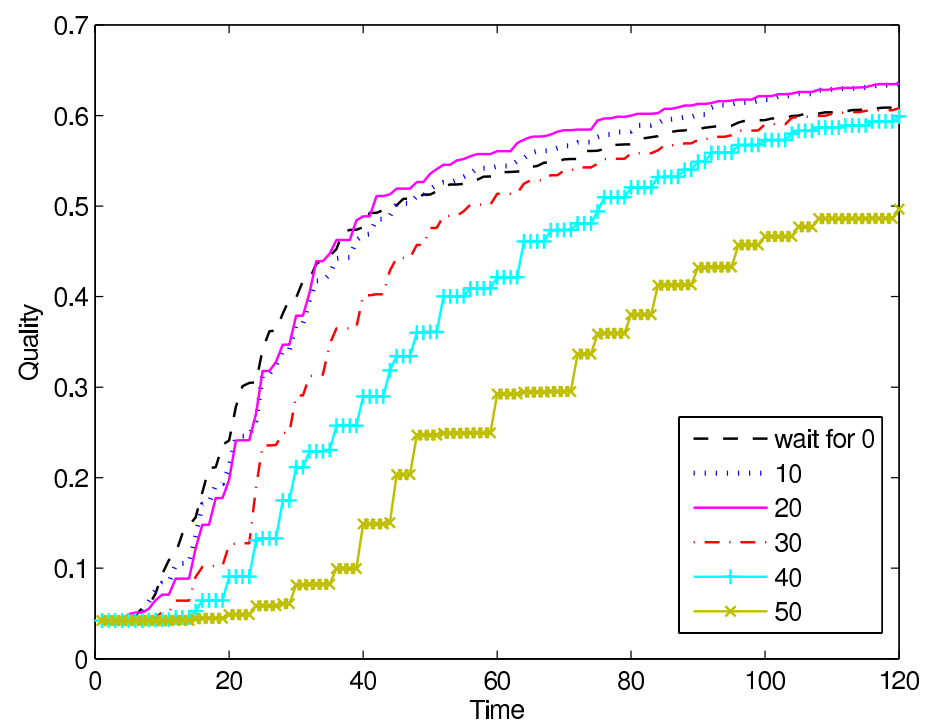

Fig. 3 The quality of the solutions obtained over time. The plots show the quality if the computing resources wait for at least $0,10,20,30,40$ and 50 other computing resources to continue the optimisation. A long waiting time corresponds to the worst quality, whereas waiting time of less than 20 percent is shown to increase the quality.

a large number of other resources means that the fast processors stay idle for a long time which is, on the other hand, undesirable when allowed only a fixed time for the entire optimisation process (such as when using the grid).

\section{Unreliable Distributed Environments}

The experiments described in the previous section demonstrated the improvements achievable using asynchronous updates in an environment consisting of heterogeneous computing resources. Parallel MOPSO was based on the straightforward Master-Slave parallelisation paradigm. In this section we seek to extend the algorithm to use in a decentralised distributed computing environment. At the time of writing, the only studies on asynchronous particle updates in parallel particle swarm algorithms have focused mainly on parallel efficiency [26, 45], without thoroughly examining the impact of parallel updates on algorithm convergence time, and without looking at the impact of evaluation failure. Failure is an important factor to consider, as it is practically unavoidable in most environments, whether caused by inter-node communication breakdowns, node failure, or even failure of the solver itself. Indeed, in distributed computing environments it is prudent to assume it is 
not a matter of if failures of computers or communications will occur, but when. The work described in this section examines the effects of asynchronous updates in distributed MOPSO algorithms in fault-prone environments (Parallel Asynchronous MOPSO), and compares the convergence characteristics of Synchronous and Asynchronous MOPSO against both iteration count and wall-clock execution time.

\section{Distributed Particle Swarm Optimisation}

As described earlier, the simplest and most common distributed particle swarm optimisation algorithms utilise a master-slave architecture, where a single controlling (master) processor runs only the optimisation algorithm, and utilises external (slave) processors to compute potential solutions [32]. Care must be taken, however, in translating this model to an unreliable distributed computing environment, as failures may impact the master node itself.

In a synchronous MOPSO, the algorithm will wait for all particles to be solved before updating particle velocities and positions using equations 1 and 2 . This allows the term iteration to be easily defined as one step of the algorithm in which all particles are evaluated. In asynchronous MOPSO, however, the algorithm will wait for only a certain proportion of the particle solutions to be returned before updates are carried out, making an iteration harder to define. For the purposes of comparing convergence against iterations completed, a single iteration of a asynchronous algorithm will be defined as a period in which the number of evaluations performed equals the number of particles in the population.

The performance of synchronous algorithms will be seriously impacted if the computing resources are not homogeneous. If some particles take longer to compute than others, at the end of each iteration most nodes in the cluster will be idle, reducing parallel efficiency, and increasing the execution time of the algorithm. Worse still, if one or more particle solutions are not returned in a timely fashion (in which case they can be said to have failed), those particles must be evaluated again, further decreasing parallel efficiency and negatively impacting the time taken to arrive at the optimal solution. The asynchronous algorithms will not suffer as significantly from these issues. A $1 \%$ synchronous environment (i.e., fully asynchronous, the algorithm does not wait for any other evaluations to be completed) asynchronous MOPSO will theoretically obtain near $100 \%$ parallel efficiency, and the impact of failures will be lessened, as a failed particle will not delay any others. In a 50\% synchronous environment, the asynchronous MOPSO will still have a somewhat higher parallel efficiency than the synchronous one, and will only be impacted by failures if half or more of the particles fail to evaluate.

However, it can be expected that the parallel asynchronous MOPSO will have some disadvantages compared to the synchronous one in regards to convergence against iterations completed. Whereas the sequential asynchronous MOPSO yielded convergence improvements over the synchronous MOPSO, the parallel synchronous MOPSO will have somewhat decreased performance. The cause of this becomes 
obvious when examining the amount of information (previous solutions) available when any given particle is updated. In the sequential asynchronous MOPSO, the number of previous solutions $P S_{s a}$ for particle $j$ at iteration $i$ for a swarm of size $N$ is given by Equation 3.

$$
P S_{s a}=(i-1) \times N+(j-1)
$$

For example, on the second $(i=2)$ iteration of a $N=100$ MOPSO, particle $j=$ 50 will have 149 previous solutions available to it. This is more than would be available to a synchronous MOPSO, which would only have the 100 solutions from the previous iteration available for the same example, as given by Equation 4.

$$
P S_{p s}=(i-1) \times N
$$

Calculating the amount of information available to a purely asynchronous MOPSO is slightly more complex, being based somewhat on when the particle evaluation is completed, but for a particle having around average evaluation time, it can be approximated as in Equation 5.

$$
P S_{p a} \simeq(i-1) \times N-\frac{N}{2}
$$

Unlike the sequential asynchronous and synchronous MOPSO, the Parallel asynchronous MOPSO at iteration $i=2$ will not usually have all the information from the previous iteration available to it, as many particles will still be under evaluation. As such, for the example used previously, a particle on the second iteration will only have, on average, 50 previous solutions available to them. This clearly suggests that, in terms of iterations completed, the parallel asynchronous MOPSO should converge more slowly than the synchronous one. It is expected, however, that this performance decrease will be countered somewhat by the increased parallel efficiency of the asynchronous MOPSO, which will allow particle evaluations (and thus iterations) to be completed more quickly than in the synchronous one.

\subsection{Simulation Setup and Testing Procedure}

In order to examine the performance of the parallel asynchronous and synchronous MOPSO in heterogeneous, fault-prone conditions, a distributed particle swarm simulation environment was constructed using both 100 particles and 100 computational nodes. A MOPSO algorithm was used, with $P_{\text {global }}$ and $P_{\text {best }}$ being chosen at random from the non-dominated sets of solutions found by the entire population and by the individual particles, respectively.

Particle evaluation times were controlled so as to conform to a normal distribution, with a mean execution time of $\mu=5$ seconds and a standard deviation $\sigma=1$ second. Modeling the evaluation times in this way meant that failures could be de- 
tected by monitoring the time taken by each solver. If a solution was not returned with $\mu+4 \sigma$ seconds, it was assumed that the solver had failed and required that the evaluation be repeated. This is a safe assumption, as (from the properties of the normal distribution) $99.997 \%$ of evaluations would have completed in this allowed time.

The solver used was the analytical ZDT1 test function described earlier. Simulations were run for varying degrees of synchronous behaviour, ranging from $1 \%$ (fully asynchronous) through to $100 \%$ (fully synchronous), and for failure rates of $0 \%, 5 \%, 10 \%$ and $20 \%$. It should be noted that these failure rates are mean failure rates, as the probability of an evaluation failure was based on a normal distribution. Results were averaged over fifteen runs to ensure reliable, repeatable experimental data was obtained. A convergence factor metric was used which measured area of the potential solution space not dominated by an approximation to the Paretooptimal front as a ratio of the solution space not dominated by the actual Paretofront. This convergence metric is given mathematically as

$$
\text { ConvergenceFactor }=\frac{P A_{\text {approximate }}}{P A_{\text {actual }}}
$$

where $P A_{\text {approximate }}$ is the non-dominated area under the approximate Pareto-optimal front, and $P A_{\text {actual }}$ is non-dominated area under the real Pareto-optimal front, which can be derived mathematically for the test functions used. An approximation to the Pareto-optimal front that is equal to the actual Pareto-front has a convergence factor of one, and a solution set that has not converged will have a convergence factor less than one (but greater than zero). This convergence factor was monitored as a function of both iterations completed and time elapsed.

In addition to node failure, grid (or node) diversity will also impact the performance of the MOPSO variants. In a more diverse grid, solution times will vary more widely, increasing the parallel efficiency advantage of the asynchronous algorithm. Conversely, a grid containing mostly homogeneous nodes will lead to very similar solution times, decreasing the parallel efficiency advantage of the asynchronous method, making a more synchronous approach desirable. To test this hypothesis, simulations were performed with varying execution time distributions for both the fully synchronous and fully asynchronous implementations.

\subsection{Results}

The first test carried out involved measuring the convergence of the algorithms as a function of iterations completed in a fault-free environment, in order to confirm the previously stated hypothesis that the more asynchronous algorithm variations will take more iterations to converge to the same point as the less asynchronous algorithms. The average convergence factor for $1 \%$ synchronous, $30 \%$ synchronous, $70 \%$ synchronous and $100 \%$ synchronous MOPSOs over 100 iterations is shown in Figure 4. 


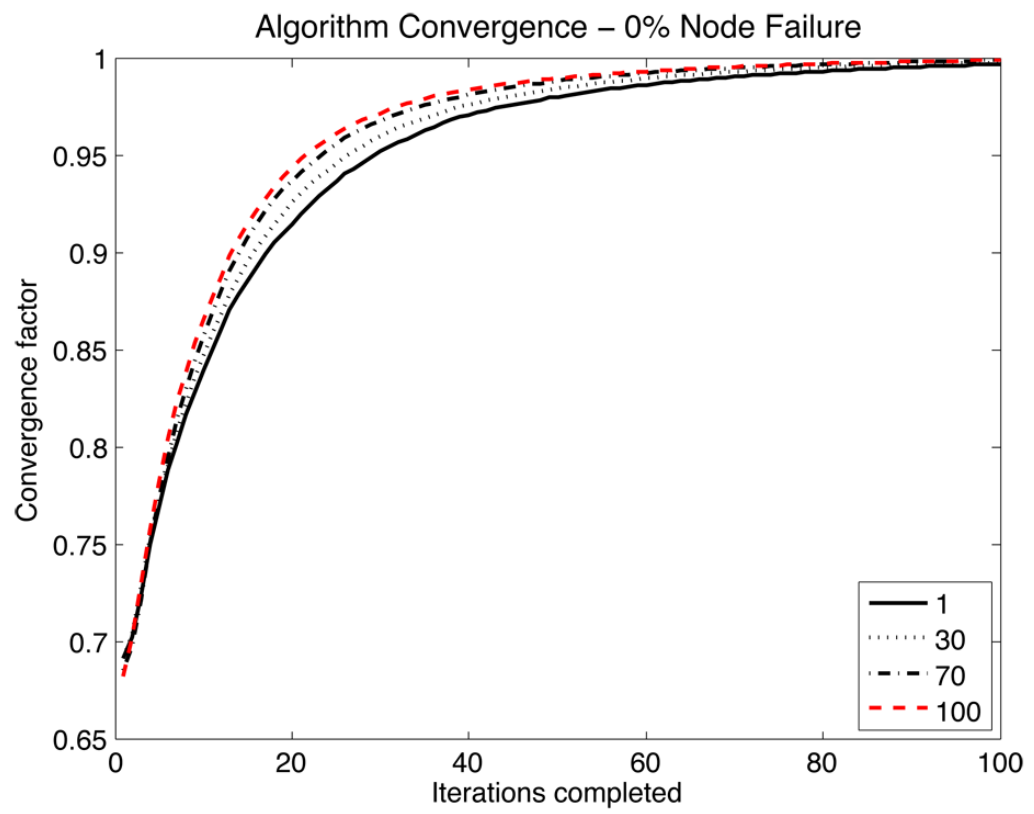

Fig. 4 Algorithm convergence for varying degrees of synchronous behaviour - 1\% (fully asynchronous), $30 \%, 70 \%$ and $100 \%$ (fully synchronous) - as a function of iterations completed, with $0 \%$ failure rate. (c)2008 IEEE

These results support the stated hypothesis, clearly showing that the fully synchronous algorithm (the parallel synchronous MOPSO) converges faster with respect to iterations completed than the asynchronous MOPSO variations, with the purely asynchronous algorithm performing the worst of all, as expected. The more synchronous the algorithm is, the faster it converges with respect to iterations completed.

Figure 5 (a) shows the algorithm convergence as a function of time for an execution time distribution having a 5\% standard deviation, representing a mostly homogeneous grid environment. In such an environment it can be seen that the synchronous update mechanism outperforms the asynchronous one, due to the reduced parallel efficiency advantage gained through the use of asynchronous updates. At some solver execution time distribution, the performance of the different update mechanisms can be expected to be approximately equal. When grid diversity is increased passed this critical point, the parallel efficiency advantage provided by asynchronous particle updates outweighs the iteration-wise convergence increase of the synchronous algorithm. This is confirmed in Figure 5 (b), which shows the convergence rate of the two algorithm variations when the distribution of solver execution times has a standard deviation of $20 \%$.

The introduction of faults has very little effect on the asynchronous (1\%, 30\% and $70 \%$ ) algorithms, whereas the performance of the synchronous algorithm drops 


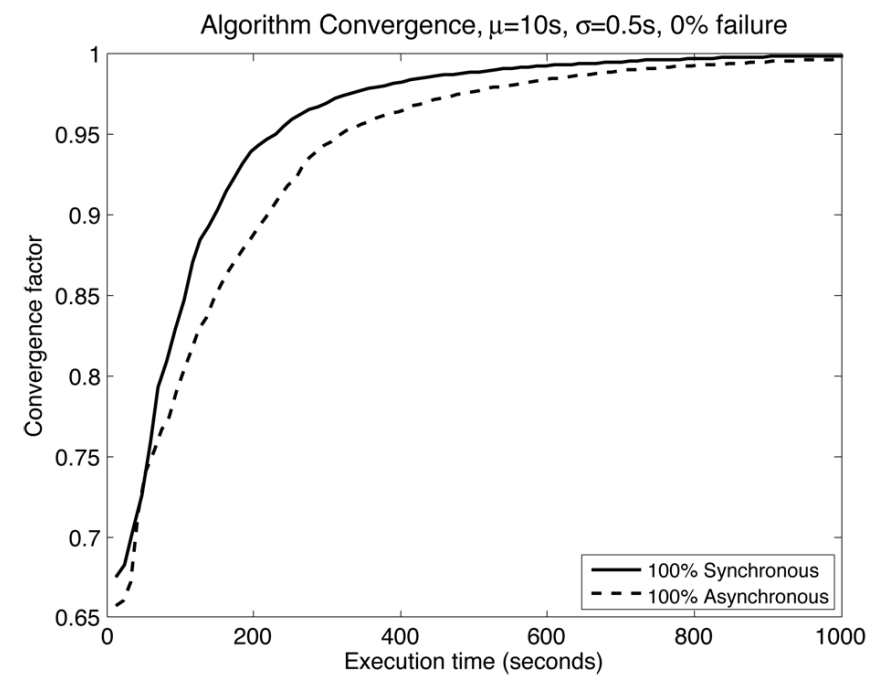

(a) $5 \%$ standard deviation in solver execution time

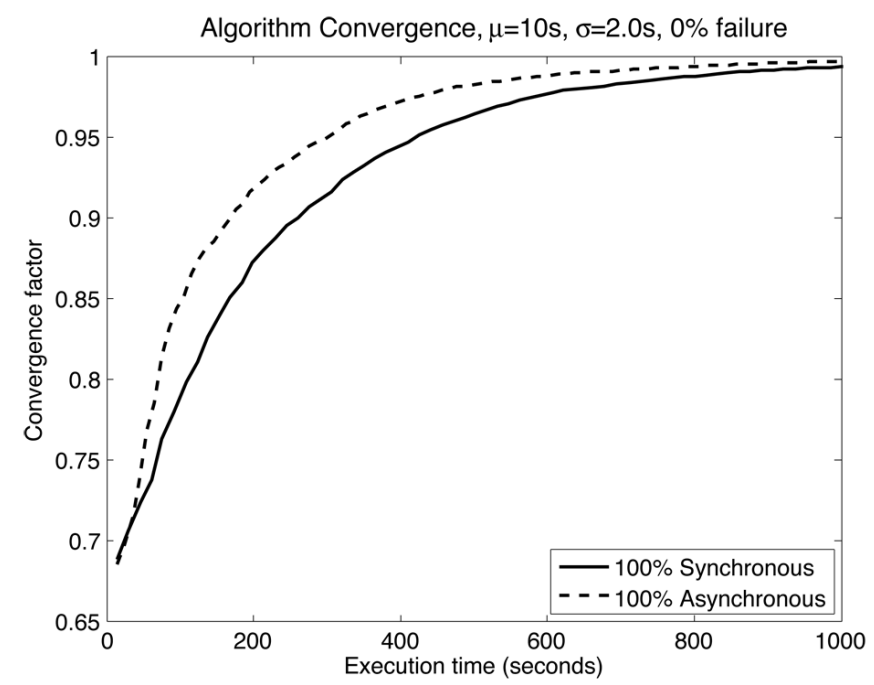

(b) $20 \%$ standard deviation in solver execution time

Fig. 5 Algorithm convergence for both completely synchronous and asynchronous behaviour

markedly. Figure 6 shows the same algorithm performance measure for a probability of failure of $10 \%$. Once the failure rate reaches $10 \%$, the $90 \%$ synchronous MOPSO's performance also starts to degrade. With increasing failure rates, the synchronous MOPSO degrades further. This extra drop in performance can be attributed 


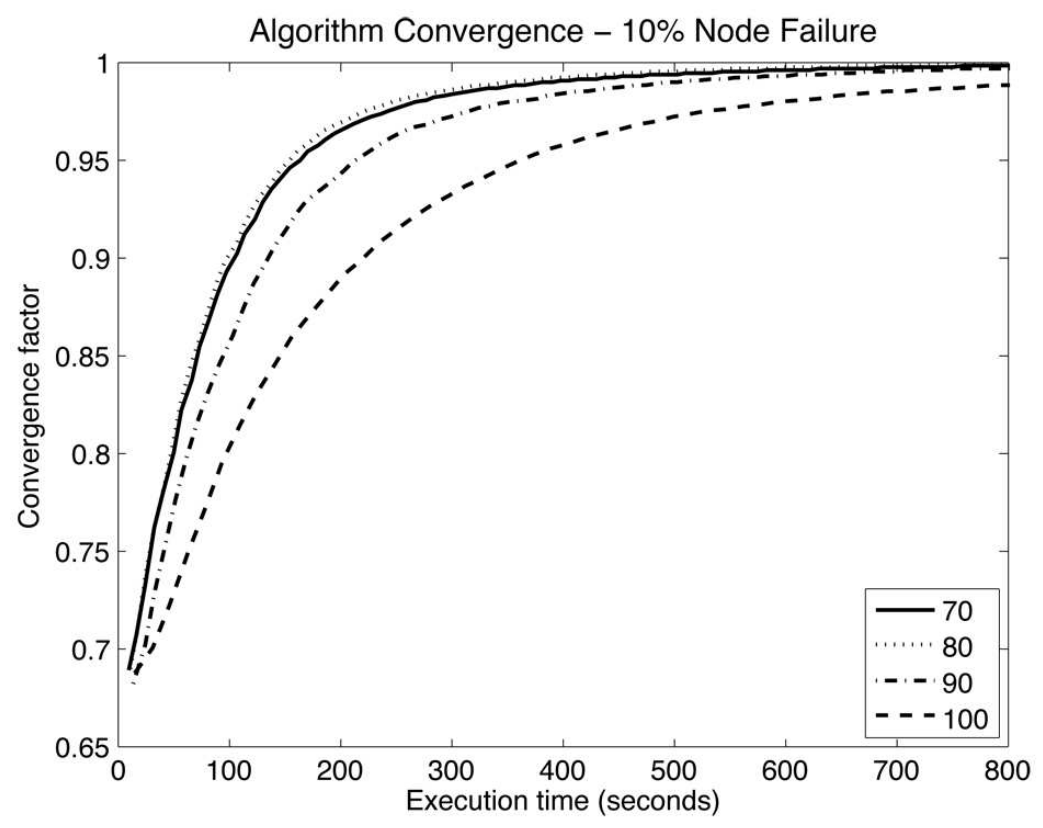

Fig. 6 Algorithm convergence for low degrees of asynchronous behaviour - 70\%, 80\%, 90\% and $100 \%$ (fully synchronous) - as a function of algorithm execution time, with $10 \%$ failure rate. (c) 2008 IEEE

to particles failing multiple times in the same iteration, further increasing the time required for each iteration.

The performance losses shown in the synchronous MOPSO and the more asynchronous MOPSO algorithms would not be evident on graphs displaying algorithm convergence as a function of completed algorithm iterations, highlighting the significance of examining the execution time of such parallel algorithms in heterogeneous, fault-prone environments.

\section{Addressing Churn}

To this point, the analysis of the algorithms has focused on their performance in the presence of failures. In a distributed computing environment, it may be the case that additional nodes will become available for use. New nodes may appear either by addition to the resources, particularly in instances in which the computational resources comprise an ad hoc grid in a peer-to-peer environment [41, 39], or by a node which previously failed returning to operation. Together with failures, the turnover in resources is collectively known as churn. When additional resources 
appear, the optimisation algorithm must decide how best to use them, generating new particles for them to evaluate.

The generation of new particle positions is a new problem which required investigation. Particle generation during the initialisation stage of evolutionary optimisation algorithms has been investigated, with the general opinion tending towards random initialisation of particles when no information on the problem space is available [47]. Some studies, however, have shown that with some prior knowledge of the problem space, intelligent positioning of the initial population leads to faster convergence to the optimal solutions [34, 4, 15, 33]. When generating new particles during the optimisation process, significant information regarding both the problem space and the current swarm location will be available, and this chapter investigates a number of methods of utilising this information to improve convergence speeds in unreliable distributed environments.

\subsection{Proposed Approaches}

Mid-execution of a particle swarm optimisation process, the algorithm has access to the current approximate to the Pareto-optimal front (that is, the best solutions found by the algorithm to that point in time). Using this information, new particle positions can be generated with aim to improve the Pareto front, by either attempting to fill gaps in it, or by extending its edges.

\subsubsection{Approach 1: Filling Gaps in the Pareto Front}

The governing equations of the particle swarm algorithm, neglecting the inertia term, cause particles to search between two points in the search space - the local and global guide solutions. Using a similar approach when generating new particles is therefore an obvious choice. Instead of using local and global guides (the former being unavailable as we are generating a new particle), the two locations to search between can be taken as the two bounding solutions in the largest gap in the current Pareto-front, for example solutions $\mathbf{x}^{\prime}$ and $\mathbf{x}^{\prime \prime}$ in Figure 7.

Once the bounding solutions are selected, each parameter $x_{k}$ is calculated for the new particle using equation 7 .

$$
x_{k}=\frac{r_{1} x_{k}^{\prime}+r_{2} x_{k}^{\prime \prime}}{r_{1}+r_{2}}
$$

The symbols $r_{1}$ and $r_{2}$ in (7) represent uniformly distributed random numbers between zero and one. Given the assumption of correlation between parameter and objective space (which is inherent in the particle swarm method), the newly generated particle should fall roughly between the two bounding solutions in the objective space. 


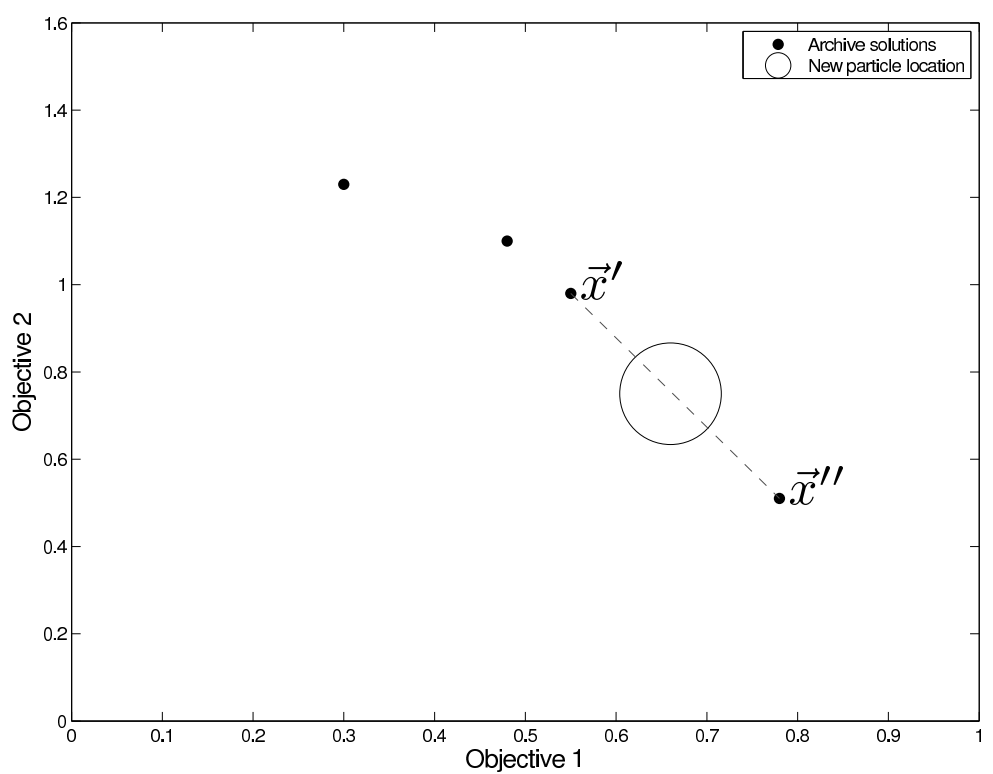

Fig. 7 Generating a new particle location by filling the largest gap in the pareto front

\subsubsection{Approach 2: Extending the Edges of the Pareto Front}

The gap-filling approach may cause the swarm to cluster towards the centre of the Pareto front, as it will never generate new particles outside the bounds of the current solutions on the edge of the front. In order to extend the edges of the front, a different approach must be used. The edge points are defined by sorting on an objective and choosing the extremes. Once the extremal solutions are selected, each parameter $x_{k}$ is calculated for the new particle using equation 8 .

$$
x_{k}=\frac{r_{1} x_{k}^{\prime}+r_{2}\left(x_{k}^{\prime}+\left(x_{k}^{\prime}-x_{k}^{\prime \prime}\right)\right)}{r_{1}+r_{2}}
$$

The symbols $r_{1}$ and $r_{2}$ in (8) again represent uniformly distributed random numbers between zero and one. The newly generated particle will this time be positioned past the current edges of the Pareto front in the solution space, again assuming correlation between the parameter and objective spaces. This approach is shown in Figure 8 . 


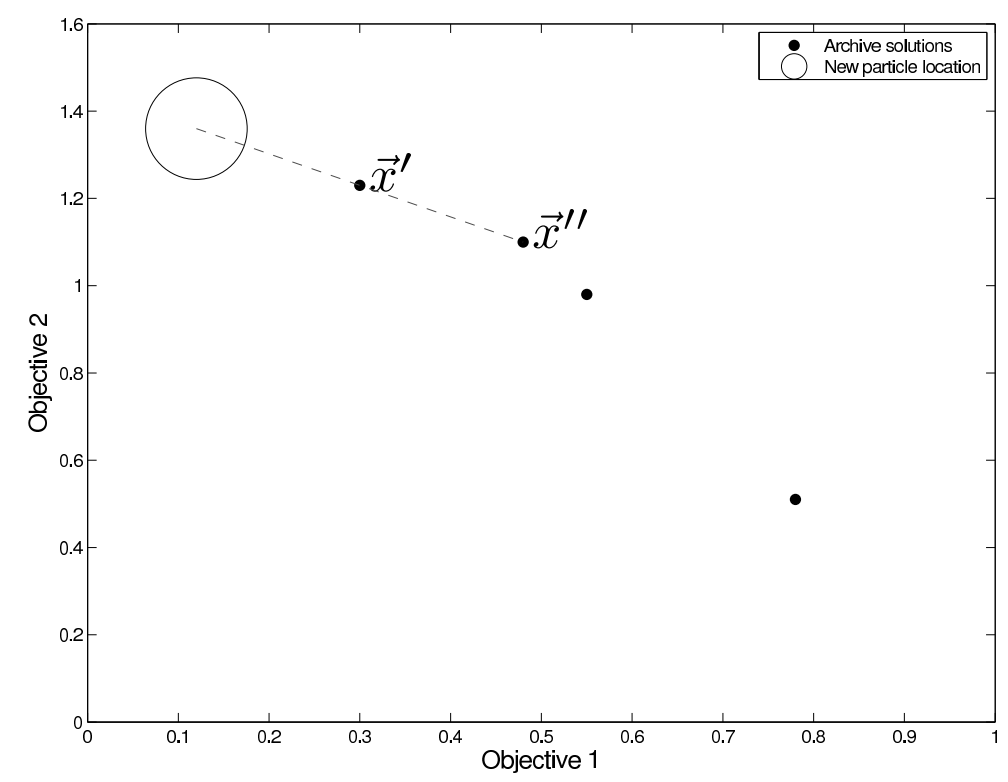

Fig. 8 Generating a new particle location by extending the edges of the Pareto front

\subsubsection{Approach 3: A Hybrid Method}

Both of the previously discussed approaches have both advantages and disadvantages. The gap filling method will promote good coverage of the global Pareto front, but only between the currently located extremal solutions. The edge extending approach, on the other hand, will promote exploration outside the current approximation to the Pareto front, but at the expense of convergence in the centre. It makes sense, then, to use a combination of the two approaches in order to gain the benefits of both while limiting the disadvantages.

\subsection{Testing Procedure}

In order to examine the performance of each approach, a testing environment was created utilising a thirty particle PSO algorithm, with one particle being removed and replaced using each of the various approaches at set intervals. Four popular analytical test functions were used as follows. Test functions ZDT1, ZDT2 and DTZL were each run for 200 iterations, with a particle replacement occurring every five iterations. The FF test function was run for 70 iterations (as it was a simpler, easier to solve problem), with particle replacement every second iteration. 
Each optimisation process was executed 200 times, with convergence and coverage metric data being averaged to give reliable results. The convergence and coverage metrics used are described later in this section. As well as the three previously described approaches, a baseline test was included where no particles were added or removed, as well as a random test where new particles were randomly generated.

\subsection{Algorithm Performance Metrics}

Up until this point, a single algorithm performance metric has been sufficient for examining the effects of altering the implementation of a distributed particle swarm optimisation algorithm. However, in order to comprehensively examine the effect of an algorithmic modification such as creating new particles during execution, two separate performance metrics are required [17]. The two metrics used are described here.

\subsubsection{Convergence Metric}

The metric used to measure algorithm convergence (the closeness of a non-dominated set to the real Pareto-optimal front) in this study does so by measuring the volume/area of the objective space which is not dominated by a given approximation to the Pareto-optimal front. This is somewhat similar to the popular hypervolume metric, which evaluates the area/volume of the objective space which is dominated by a given set of non-dominated solutions [48]. This inverted hypervolume metric was used as the lower bounds for all objective in each of the test functions used are known to be zero, providing a convenient boundary for a hypervolume-like metric. In the case of minimisation problems, such as the test functions used for this chapter, a smaller value for this metric denotes a closer approximation to the global Pareto-optimal front.

\subsubsection{Coverage Metric}

The coverage metric used for this research is a new one, as no existing metrics were deemed suitable for the purposes of this study. Existing diversity metrics [17] measure the statistical spread or evenness of the Pareto-front, rather than quantifying how well a given non-dominated set covers the objective space. An approximate front may be very evenly spread (that is, the distances between adjacent particles are relatively similar). However, it may contain very few solutions and/or not adequately cover the entire range of possible objective values. In such cases existing diversity metrics would give a misleading evaluation of the quality of the approximate Paretooptimal front. 
A new coverage metric is proposed whereby the objective space is divided into sectors originating from the utopia point. The value of the diversity metric is then given as the ratio of these sectors which contain at least one member of the nondominated set to the total number of sectors, or, mathematically:

$$
\begin{aligned}
\Psi & =\frac{1}{N} \sum_{n=1}^{N} \psi_{n} \\
\text { where } \psi_{n} & =\left\{\begin{array}{l}
1, \text { if } \exists \mathscr{S} \in \mathscr{P} \mathscr{F}, \alpha_{n-1} \leq \tan \frac{f_{1}(\mathbf{x})}{f_{2}(\mathbf{x})} \leq \alpha_{n} \\
0, \text { otherwise }
\end{array}\right.
\end{aligned}
$$

A simple example of this metric can be seen in Figure 9. In this example, the solution space is divided into ten equal segments, with seven of these segments containing solutions in the current approximation to the Pareto-optimal front. This approximate Pareto front would therefore be classified as having a 0.7 or $70 \%$ coverage factor. Practically, more segments are used to give a higher resolution result in this study, the same number of segments were used as there were particles in the swarm.

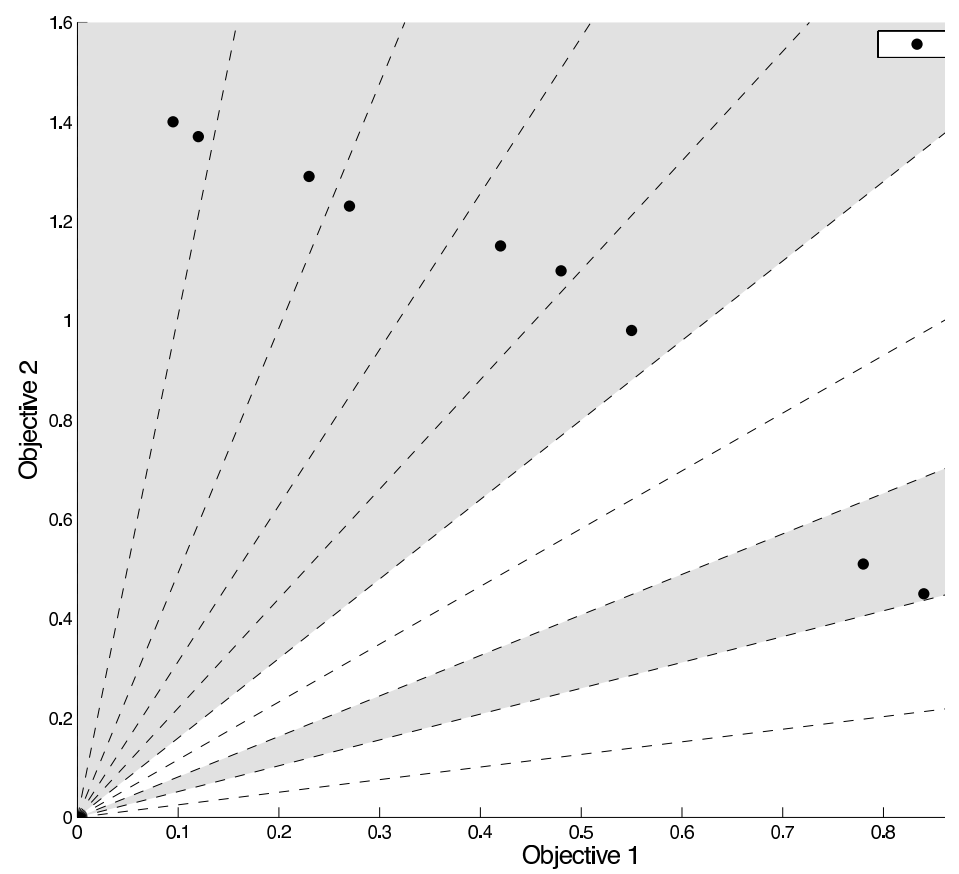

Fig. 9 Application of the proposed coverage metric to an example pareto front 

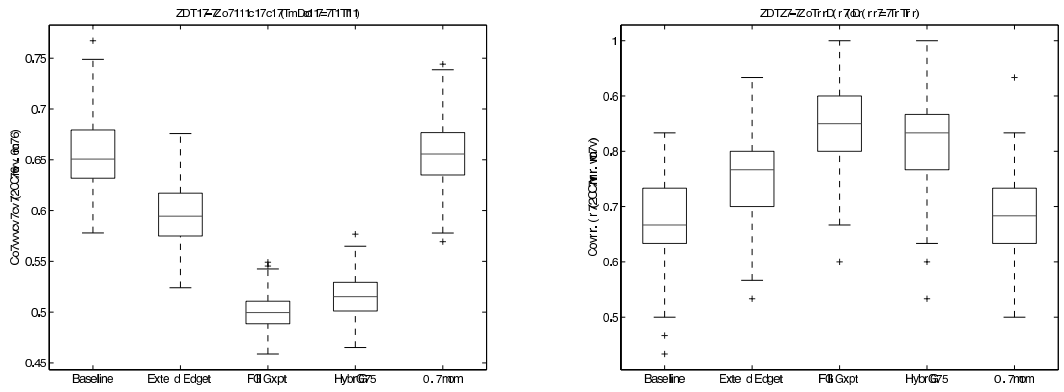

Fig. 10 Convergence (left) and coverage (right) of PSO algorithm on ZDT1 problem.
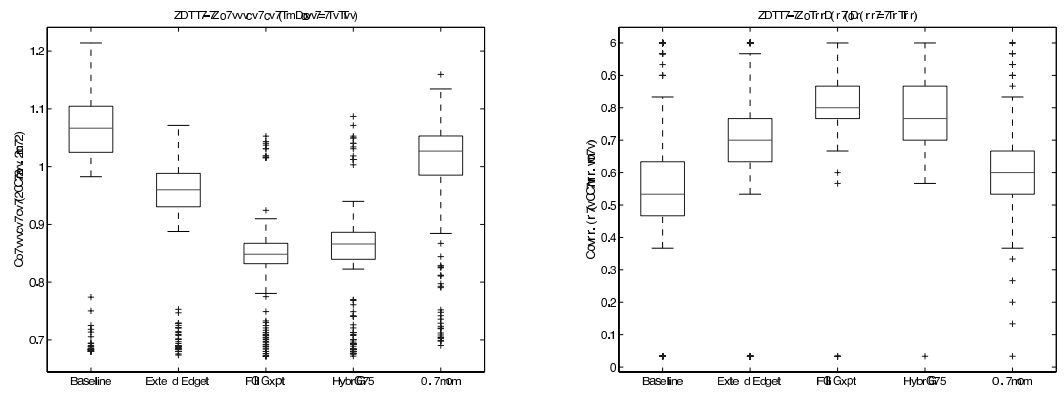

Fig. 11 Convergence (left) and coverage (right) of PSO algorithm on ZDT2 problem.

\subsection{Experimental Results}

Box plots of the convergence and coverage metrics for each test function and algorithmic approach can be seen in Figures 10 through 13.

It can be seen that the gap filling method significantly out-performs edge extending approach in both the ZDT1 and ZDT2 test functions, in terms of both convergence and coverage of the Pareto-optimal front. Both approaches appear to result in better optimisation results than the baseline algorithm and the random generation method.

The two approaches faired slightly differently when applied to the FF test function. The gap filling method still resulting in increased algorithm convergence when compared to extending the edges, however the difference between the two approaches here was significantly less than for the ZDT functions. Unlike the results for two ZDT functions, here the edge extending approach achieves, on average, better coverage of the Pareto front than the gap filling method for generating new particles. 

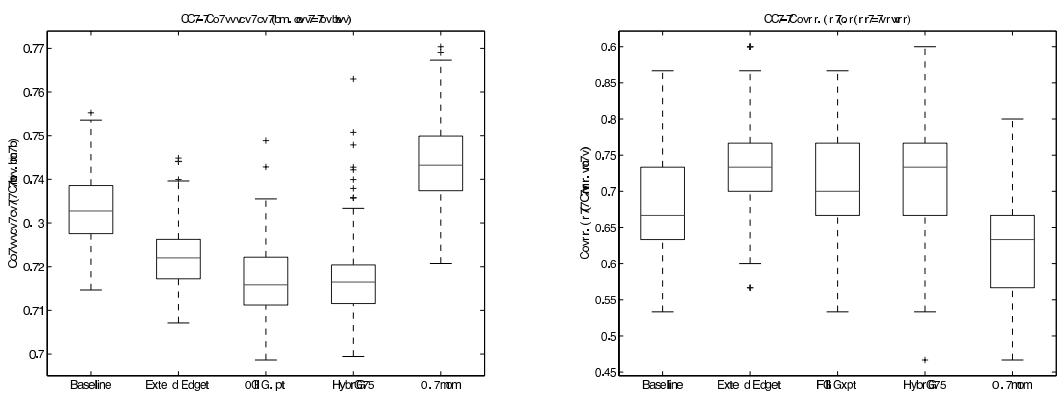

Fig. 12 Convergence (left) and coverage (right) of PSO algorithm on FF problem.
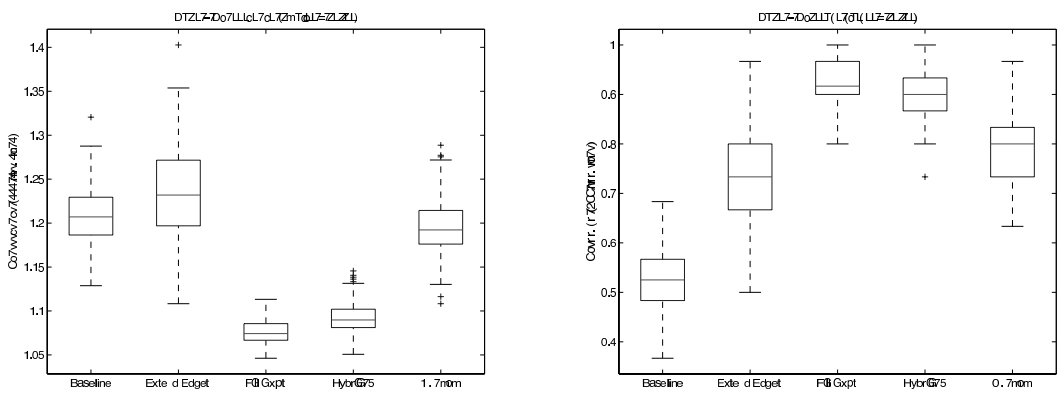

Fig. 13 Convergence (left) and coverage (right) of PSO algorithm on DTZL problem.

The results for the DTZL test function are comparable to those obtained using the ZDT1 and ZDT2 functions, with the gap filling approach being the top performer.

The hybrid approach, which generated new particles using the gap filling approach $75 \%$ of the time and used the edge extending approach the other $25 \%$, can be seen to give the most consistent results across all four test functions. In all cases, the results produced by the hybrid approach compare quite favourable with those of the top performing method.

A question remains as to how well the methods described in this section scale with increased numbers of objectives, i.e., an objective space of higher dimensionality. When finding gaps, it is not difficult to generalise the method to higher dimensions. It suffices to find the nearest neighbour for each point in the set of nondominated solutions by means of calculating the Euclidian distance between them in objective space, and select the pair for which this distance is a maximum. This operation has a computational complexity of $O\left((N / 2)^{2}\right)$ but, given the assumption that the evaluation of objective functions dominates computation time, this can be ignored. Extending edges is less simple: as the dimensionality of objective space increases, so do the number of edges to be considered and it is not clear that the function of the algorithm will be best served by choosing only extremal points. The 
effects of higher dimensionality on algorithm performance remain a subject for future work.

With regard to the coverage metric, the metric operates essentially by taking an $N-1$ dimensional "slice" through the objective space, trivially extensible to multiple dimensions. As, however, this effectively collapses the distribution of the nondominated set into a projection onto a single dimension, care should be exercised interpreting results for higher-dimensional problems.

\section{Conclusions}

In this chapter, we studied the Master-Slave model of parallelisation to solve multiobjective optimisation problems on a heterogeneous set of resources. We proposed a new hybrid Parallel MOPSO method called PMOPSO which uses a Gap Search method to search unexplored regions of the parameter space. PMOPSO was tested on a scenario containing a set of heterogeneous resources and compared with 3 other cases. The results show a good quality of solutions even when compared with a non-parallel case which takes a longer computation time. The proposed approach is particularly suitable for very expensive multi-objective problems of real-world applications using a heterogeneous set of processors.

Consideration of asynchronous update in parallel MOPSO algorithms was extended from the Master-Slave model to a general, distributed computing approach. From the results presented in this chapter it can be concluded that asynchronous particle updates, while negatively impacting algorithm convergence with respect to iterations completed, allow parallel distributed particle swarm algorithms to gracefully and efficiently handle node and solver heterogeneity as well as node, network and solver failure. Using asynchronous particle updates has been shown to increase parallel efficiency [26], which in turn decreases the computation time required for each algorithm iteration. This decrease in iteration time mitigates the decrease in algorithm convergence as a function of iterations completed which is introduced by the asynchronous update mechanism.

Simulations performed have shown that the more likely failures are, the more asynchronous the parallel particle swarm algorithm should be made. The degree of grid heterogeneity will need to be considered alongside grid volatility when setting the degree of synchronisation in particle updates. In fairly reliable environments such as fixed, dedicated high-performance computer clusters, mostly synchronous algorithms can be used, however if deploying into more volatile environments, such as distributed computer grids or non-dedicated peer-to-peer systems, the algorithms used should be made more asynchronous to counter the higher number of failures that can be expected. The degree of synchronisation could be set dynamically, allowing the performance of the algorithm to be maintained in dynamic grid environments.

Finally, we considered the issue of churn, in which compute resources may not only disappear through failure, but appear, by a variety of mechanisms, to take on 
tasks. Two methods, Pareto front gap filling and edge extending, and proposed a hybrid approach were evaluated for their ability to fully and effectively utilise available resources in decentralised, fault-prone distributed environments. The proposed approaches were tested using a number of popular analytical optimisation test functions, and performance evaluated with convergence and coverage metrics. Generating new particles by attempting to fill gaps in the Pareto-optimal front generally performed better than extending the front's edges. However it was shown that extending the edges of the Pareto-front can be more advantageous in certain scenarios. From this, a hybridised method was formulated to utilise both approaches while favouring gap filling, and this hybrid method was shown to perform consistently well across all problems used for testing.

Results presented here have shown that asynchronous updates in parallel particle swarm algorithms can lead to significant performance increases in diverse grid environments, where solver execution times will vary widely. Future work is desirable to investigate dynamic methods to respond to changing computing environments, in both the degree of synchronisation and manner in which additional compute resources are utilised. In addition, further analysis of the performance of PAPSO algorithms on other test problems with higher numbers of parameters and objectives is required.

Acknowledgements The authors would like to thank Jürgen Branke for his contributions to the early stages of this work. Some portions of the work described in this chapter are reprinted, with permission, from Scriven, I., Ireland, D., Lewis, A., Mostaghim, S. and Branke, J., "Asynchronous Multiple Objective Particle Swarm Optimisation in Unreliable Distributed Environments" and Mostaghim, S., Branke, J., Lewis, A. and Schmeck, H., "Parallel Multi-objective Optimization Using Master-slave Model on Heterogeneous Resources", in WCCI 2008 Proceedings (2008 IEEE World Congress on Computational Intelligence) (C)2008 IEEE.

\section{References}

[1] Abramson D, Lewis A, Peachy T (2000) Nimrod/O: A tool for automatic design optimization. In: The 4th International Conference on Algorithms and Architectures for Parallel Processing (ICA3PP 2000)

[2] Al-Kazemi B, Mohan C (2002) Multi-phase generalization of the particle swarm optimization algorithm. In: Proceedings of the IEEE Congress on Evolutionary Computation 2002, vol 2, pp 1057-1062

[3] Alba E, Tomassini M (2002) Parallelism and evolutionary algorithms. IEEE Transactions on Evolutionary Computation 6(5):443-461

[4] Angeline PJ (1998) Evolutionary optimization versus particle swarm optimization: Philosophy and performance differences. In: Evolutionary Programming VII, Springer Verlag, Lecture Notes in Computer Science, vol 1447, pp 601610

[5] Branke J, Mostaghim S (2006) About selecting the personal best in multiobjective particle swarm optimization. In: et al TPR (ed) Parallel Problem 
Solving from Nature, Springer, LNCS, pp 523-532

[6] Branke J, Kamper A, Schmeck H (2004) Distribution of evolutionary algorithms in heterogeneous networks. In: Genetic and Evolutionary Computation Conference, Springer, LNCS, vol 3102, pp 923-934

[7] Branke J, Schmeck H, Deb K, Reddy M (2004) Parallelizing Multi-Objective Evolutionary Algorithms: Cone Separation. In: IEEE Congress on Evolutionary Computation, pp 1952-1957

[8] Branke J, Deb K, Miettinen K, Slowinski R (2008) Multiobjective Optimization Interactive and Evolutionary Approaches. Springer Verlag

[9] Bui LT, Abbass HA, Essam D (2007) Local models - an approach to distributed multiobjective optimization. Journal of Computational Optimization and Applications

[10] Cantu-Paz E (1997) Designing efficient master-slave parallel genetic algorithms. IlliGAL Report 97004, University of Illinois

[11] Cantu-Paz E (1997) A survey of parallel genetic algorithms. IlliGAL Report 97003, University of Illinois

[12] Cantu-Paz E (2000) Efficient and Accurate Parallel Genetic Algorithms. Kluwer

[13] Carlisle A, Dozier G (2001) An off-the-shelf PSO. In: Proceedings of the 2001 Workshop in Particle Swarm Optimisation, pp 1-6

[14] Censor Y, Zenios SA (1997) Parallel Optimization: Theory, Algorithms, and Applications. Oxford University Press, New York/Oxford

[15] Chou CH, Chen JN (2000) Genetic algorithms: initialization schemes and genes extraction. In: Fuzzy Systems, 2000. FUZZ IEEE 2000. The Ninth IEEE International Conference on, vol 2, pp 965-968

[16] Coello CAC, Veldhuizen DAV, Lamont GB (2002) Evolutionary Algorithms for Solving Multi-Objective Problems. Kluwer Academic Publishers

[17] Deb K, Agrawal S, Pratap A, Meyarivan T (2000) A fast elitist non-dominated sorting genetic algorithm for multi-objective optimization: NSGA-II. In: Parallel Problem Solving from Nature VI (PPSN-VI), pp 849-858

[18] Deb K, Thiele L, Laumanns M, Zitzler E (2002) Scalable multi-objective optimization test problems. In: Congress on Evolutionary Computation, IEEE, pp 825-830

[19] Deb K, Zope P, Jain A (2003) Distributed computing of pareto-optimal solutions with evolutionary algorithms. In: International Conference on Evolutionary Multi-Criterion Optimization, pp 534-549

[20] Fonseca CM, Fleming PJ (1996) On the performance assessment and comparison of stochastic multiobjective optimizers. In: Parallel Problem Solving from Nature (PPSN IV), Lecture Notes in Computer Science, pp 584-593

[21] Goldberg DE (1989) Genetic Algorithms in Search, Optimization and Machine Learning. Addison-Wesley Publishing Company, Inc., Reading, Massachusetts

[22] Gupta I, Ganesh AJ, Kermarrec AM (2006) Efficient and adaptive epidemicstyle protocols for reliable and scalable multicast. IEEE Transactions on Parallel and Distributed Systems 17(7):593-605 
[23] Hughes EJ (2003) Multi-objective binary search optimisation. In: Evolutionary Multi-Criterion Optimization: Second International Conference (EMO), pp 72-87

[24] Kennedy J, Eberhart RC (2001) Swarm Intelligence. Morgan Kaufmann

[25] Knowles J (2005) A summary-attainment-surface plotting method for visualizing the performance of stochastic multiobjective optimizers. IEEE Intelligent Systems Design and Applications (ISDA V)

[26] Koh B, George AD, Haftka RT, Fregly BJ (2006) Parallel asynchronous particle swarm optimisation. International Journal for Numerical Methods in Engineering 67(4):578-595

[27] Laredo JLJ, Castillo PA, Mora AM, Merelo JJ (2008) Evolvable agents, a fine grained approach for distributed evolutionary computing: walking towards the peer-to-peer computing frontiers. Soft Computing 12(12):1145-1156

[28] Laredo JLJ, Eiben AE, van Steen M, Merelo JJ (2008) On the run-time dynamics of a peer-to-peer evolutionary algorithm. In: Parallel Problem Solving from Nature X PPSN, pp 236-245

[29] Mostaghim S, Teich J (2003) Strategies for finding good local guides in multiobjective particle swarm optimization. In: IEEE Swarm Intelligence Symposium, pp 26-33

[30] Mostaghim S, Teich J (2004) A new approach on many objective diversity measurement. In: Dagstuhl Proceedings number 04461, Dagstuhl, Germany

[31] Mostaghim S, Teich J (2005) A new approach on many objective diversity measure. In: Proceedings of the Dagstuhl Seminar 04461

[32] Mostaghim S, Branke J, Schmeck H (2007) Multi-objective particle swarm optimization on computer grids. In: The Genetic and Evolutionary Computation Conference, vol 1, pp 869-875

[33] Mostaghim S, Branke J, Lewis A, Schmeck H (2008) Parallel multi-objective optimization using a master-slave model on heterogeneous resources. In: IEEE, Congress on Evolutionary Computation (CEC)

[34] Rahnamayan S, Tizhoosh HR, Salama MMA (2007) A novel population initialization method for accelerating evolutionary algorithms. Comput Math Appl 53(10):1605-1614

[35] Reyes-Sierra M, Coello CAC (2006) Multi-objective particle swarm optimizers: A survey of the state-of-the-art. International Journal of Computational Intelligence Research 2(3):287-308

[36] Riget J, Vesterstrøm J (2002) A diversity-guided particle swarm optimizer the ARPSO. Technical report, University of Aarhus, Department of Computer Science

[37] Schutte JF, Reinbolt JA, Fregly BJ, Haftka RT, George AD (2004) Parallel global optimisation with particle swarm algorithm. International Journal for Numerical Methods in Engineering 61:2296-2315

[38] Scriven I, Lewis A, Ireland D, Lu J (2008) Distributed multiple objective particle swarm optimisation using peer to peer networks. In: IEEE Congress on Evolutionary Computation (CEC) 
[39] Scriven I, Lewis A, Smith M, Friese T (2008) Resource evaluation and node monitoring in service oriented ad-hoc grids. In: Proc. Sixth Australasian Symposium on Grid Computing and e-Research (AusGrid 2008), CRPIT, vol 82, pp 65-71

[40] Scriven I, Lu J, Lewis A (2008) An efficient peer-to-peer particle swarm optimiser for EMC enclosure design. In: The 13th Biennial IEEE Conference on Electromagnetic Field Computation (CEFC)

[41] Smith M, Friese T, Freisleben B (2004) Towards a service-oriented ad hoc grid. In: Proc. 3rd International Symposium on Parallel and Distributed Computing, IEEE Computer Society

[42] Talbi EG, Mostaghim S, Okabe T, Ichibushi H, Rudolph G, Coello CC (2008) Parallel Approaches for Multiobjective Optimization, Springer Verlag, pp 349-372

[43] Veldhuizen DAV, Zydallis J, Lamont GB (2003) Considerations in engineering parallel multiobjective evolutionary algorithms. In: IEEE Transactions on Evolutionary Computation, Vol. 7, No. 2, pp 144-173

[44] Venter G, Sobieszczanski J (2004) Multidisciplinary optimisation of a transport aircraft wing using particle swarm optimisation. Structural and Multidisciplinary optimisation 26(1-2):121-131

[45] Venter G, Sobieszczanski-Sobieski J (2006) A parallel particle swarm optimisation algorithm accelerated by asynchronous evaluations. Journal of Aerospace Computing, Information, and Communication 3(3):123-137

[46] Wickramasinghe WRMUK, van Steen M, Eiben AE (2007) Peer-to-peer evolutionary algorithms with adaptive autonomous selection. In: Proceedings of the Genetic and Evolutionary Computation Conference (GECCO), pp 14601467

[47] Xie XF, Zhang WJ, Yang ZL (2002) Adaptive particle swarm optimization on individual level. In: Signal Processing, 2002 6th International Conference on, vol 2, pp 1215-1218

[48] Zitzler E (1999) Evolutionary Algorithms for Multiobjective Optimization: Methods and Applications. Shaker 